\title{
Multiple Comparison Procedures for Simple One-Way ANOVA with Dependent Data
}

\author{
Guillermo Vallejo Seco, Ignacio Menéndez de la Fuente, and Paula Fernández García \\ University of Oviedo
}

The independence assumption, although reasonable when examining cross-sectional data using single-factor experimental designs, is seldom verified by investigators. A Monte Carlo type simulation experiment was designed to examine the relationship between true Types I and II error probabilities in six multiple comparison procedures. Various aspects, such as patterns of means, types of hypotheses, and degree of dependence of the observations, were taken into account. Results show that, if independence is violated, none of the procedures control a using the error rate per comparison. At the same time, as the correlation increases, so does the per-comparison power.

Key words: error rate per comparison, per-pairs power, dependent data

\begin{abstract}
La asunción de independencia parece un supuesto razonable al examinar los datos de un diseño experimental de grupos al azar. Probablemente debido a ello, esta asunción raramente es verificada por los investigadores. Por todo ello, realizamos un experimento de simulación Monte Carlo por medio del cual se examinan las tasas de error tipo I y tipo II cometidas al utitizar diferentes procedimientos de comparación múttiple, haciendo uso de diferentes tipos de patrones de medias, tipos de hipótesis y grados de dependencia entre las observaciones. Si se viola la independencia, los resultados revelan que ningún procedimiento mantiene controlada la tasa de error por contraste al nivel nominal, al mismo tiempo, conforme se incrementa la correlación en una pequeña cantidad, la potencia por comparación también se incrementa.
\end{abstract}

Palabras clave: error tipo I, potencia, comparación múltiple, Monte Carlo

This research was funded by a grant from the University of Oviedo (Rcf.: IR-95-0622-1).

Correspondence concerning this article should be addressed to Dr. Guillermo Vallejo, Departamento de Psicología. Universidad de Oviedo. Plaza Feijoo, s/n. 33003 OVIEDo. Spain. E-mail: gvallejo@sci.cpd.uniovi.es 
Many experiments have been designed to determine whether there are any treatment effects. As a general rule, when hypothesis testing leads researchers to reject a null hypothesis, they to explore the data in order to look for such effects. Various methods have been proposed for this. The choice of a procedure to compare multiple pairs of means is not a simple task; at present, there are various procedures, and the available options list continues to grow. On the other hand, we are faced with a paradox: whereas the importance of the non-fulfillment of the normality and/or homogeneity assumptions is considered relative, numerous tests are presented to prove these assumptions, including some tests that do not require their verification. With regard to this, researchers generally presume that they have enough randomly distributed units to insure the fulfillment of the independence assumption. However, experimental procedures, including randomization of subjects to tratment conditions, may not be correct (e.g., when a random sample is chosen from a small population or a study is carried out with subjects working together in groups). Whenever the treatment involves interaction among subjects, there is a smaller within-group variation than might be expected if observations were statistically independent from each other.

We proposed to test the following procedures empirically: Fisher's (1935) least significant difference procedure (LSD); Fisher (1935) and Hayter's (1986) procedure (FH); Newman (1939) and Keuls's (1952) procedure (NK); the REGW procedure by Ryan (1959), Einot and Gabriel (1975), and Welsch (1977); Tukey's (1953) honest significant difference procedure (HSD); and Scheffe's (1959) procedure (S). At the same time, we compared means under the non-fulfillment of the independence assumption with cross section data. For all this, we took into account the Type I error rate and the test power for each analysis procedure. Type I error was examined using as conceptual unit the error rate per comparison, which is defined for each hypothesis as the probability of Type I error (Shaffer, 1995). Power was examined using per-comparison power, which is the average probability of detecting a true mean difference in the $q$ means in the experiment (Einot \& Gabriel, 1975). Our second goal was to sce whether the method proposed by Pavur (1988) to correct the lack of independence is effective.

Although analytic derivation has been carried out on the subject, computer simulation has not yet been put into practice.

\section{Correcting for correlation procedure}

Consider the full rank experimental design model for balanced one-way ANOVA, comparing $q$ treatments with $n$ observations per treatment. This model can be written as:

$$
\mathbf{y}=\mathbf{X} \beta+\varepsilon,
$$

where $\mathrm{y}$ is an $N \times I$ vector of observations, $\mathrm{X}$ is an $N \times q$ between groups design matrix, $\beta$ ' is a $/ \times q$ vector of parameters $\left(\mu_{1}, \mu_{2}, \ldots, \mu_{q}\right)$, and $E$ is a $N \times /$ vector of random errors normally distributed with mean equal to $z e r o, E(\varepsilon)=$ $\mathbf{0}$, and variance equal $10 \sigma^{2} \mathrm{U}, V(\varepsilon)=\sigma_{\varepsilon}^{2} \mathbf{U}$; where $\mathrm{U}$ is a cortelation matrix and $\sigma_{\varepsilon}^{2}$ is the constant variance of the $\varepsilon$ for each group.

The correlation $\left(p_{1}\right)$ that is considered in the vector of observations used in the present research, $y^{\prime}=$ $\left[Y_{I}, Y_{I 2}, \ldots, Y_{l n}, \ldots, Y_{q l}, \ldots, Y_{q n}\right]$, is one in which the correlation of any two observations within a group is the same and the correlation between any two observations in different groups is equal to zero $\left(\rho_{2}=0\right)$. For this reason, $\mathbf{U}$ is a block diagonal matrix. The $\mathbf{U}$ matrix has the following form:

$$
\left[\begin{array}{cccc}
A & O & \ldots & \text { O } \\
\text { O } & \text { A } & \ldots & \text { O } \\
\vdots & \vdots & \vdots & \vdots \\
O & O & \ldots & A
\end{array}\right]
$$

where $\mathbf{O}$ is an $n \times n$ null matrix and $\mathbf{A}$ is an $n \times n$ matrix where all of the variances on the main diagonal are equal and all of the covariances off the main diagonal are equal; that is, it meets the compound symmetry assumption. Following the suggestions of Pavur and Lewis (1983), if we define $\mathbf{M}_{n}$ $=n^{-1} \mathbf{1}_{\mathbf{n}} \mathbf{1}_{\mathrm{n}}, \mathbf{M}_{\mathrm{q}}=q^{-1} 1_{\mathrm{q}} \mathbf{l}^{\prime}{ }_{\mathrm{q}}, \mathbf{M}_{\mathrm{nq}}=\mathbf{M}_{\mathbf{n}} \otimes \mathbf{M}_{\mathrm{q}}, \mathbf{C}_{\mathbf{n}}=\mathbf{I}_{\mathrm{n}}-\mathbf{M}_{\mathrm{n}}$, and $\mathrm{C}_{\mathrm{q}}=\mathbf{I}_{\mathrm{q}}-\mathbf{M}_{\mathrm{q}}$, the correlation matrix can be written as:

$$
\mathbf{U}=c_{1} \mathbf{C}_{\mathrm{q}} \otimes \mathbf{M}_{\mathrm{n}}+c_{2} \mathbf{I}_{\mathrm{q}} \otimes \mathbf{C}_{\mathbf{n}}+c_{3} \mathbf{M}_{\mathrm{nq}},
$$

where $c_{1}=1+\rho_{1}(\mathrm{n}-1), c_{2}=\left(1-\rho_{1}\right)$, and $c_{3}=c_{1}$. Scariano and Davenport (1987) have shown that if $\mathbf{U}=\mathbf{I}_{\mathrm{N}}$, then $c_{\xi}$ $=c_{2}=c_{3}$.

Once the conelation structure on $\mathbf{y}$ is defined, we now specify how to estimate the parameter vector $\beta$ when the errors are correlated. Aitken's (1935) generalization of the Gauss-Markov least-squares theorem established that the best estimator of $\beta$ is:

$$
\beta=\left[\mathbf{X}^{\prime} \mathbf{V}(\rho)^{-1} \mathbf{X}\right]^{-1} \mathbf{X}^{\prime} \mathbf{V}(\rho)^{-1} \mathbf{y}
$$

so as to have minimum variance among all unbiased estimators. In order to avoid operating with matrices of great dimension, in practice, it is more convenient to find a nonsingular transformation matrix, say, $\mathbf{P}$, such that:

$$
\mathbf{P}^{\prime} \mathbf{P}=\mathbf{U}^{-1} \text { and } \mathbf{P U} \mathbf{P}^{\prime}=\mathbf{I} \text {. }
$$

Generalized least squares or Aitken (1935) estimation. may then be achieved by ordinary least-squares to the original model after premultiplication by $\mathbf{P}$. That is, if the equation to be estimated is premultiplied by $\mathbf{P}$, so that $\mathbf{P y}$ $=\mathbf{P X} \beta+\mathbf{P} \varepsilon$, then ordinary least-squares applied to the 
transformed variables $(\mathbf{P y}, \mathbf{P X}$, and $\mathbf{P \varepsilon})$ is equivalent to generalized least squares. Therefore, the estimator of $\beta$ satisfies the properties of the theorem of Gauss-Markov and the error term of the transformed model has a covariance structure equal to $\sigma_{\varepsilon}^{2} I$. There are various procedures for the construction of the transformation matrix $\mathbf{P}$ (see Vallejo \& Fernández, 1990). However, the notation employed here is the one by Pavur (1988). This can be expressed as:

$\mathbf{P}=\left(\frac{1}{\sqrt{c_{1}}}\right) \mathbf{C}_{\mathrm{q}} \otimes \mathbf{M}_{\mathrm{n}}+\left(\frac{1}{\sqrt{c_{2}}}\right) \mathbf{I}_{\mathrm{q}} \otimes \mathbf{C}_{\mathrm{n} 1}+\left(\frac{1}{\sqrt{c_{3}}}\right) \mathbf{M}_{\mathrm{nq}}(6)$

Finally, is possible to calculate a $t$ statistic for any given comparison:

$$
t_{\dot{\psi}}=\frac{\hat{\Psi}_{i}}{\hat{\sigma}_{\psi_{L}}}=\frac{{\overline{Y_{k}}}^{*}-{\overline{Y_{k}}}^{*}}{\sqrt{\frac{2 M S_{\text {error }}{ }^{*}}{n}}},
$$

where $M S_{\text {error }}{ }^{*}=\left[(\mathbf{P y})^{\prime}\left(\mathbf{I}_{\mathrm{q}} \otimes \mathbf{C}_{\mathrm{n}}\right)(\mathbf{P y})\right] / \mathrm{n}$. Once the $t$ statistic is computed, it can be compared with the corresponding critical value to each one of the multiple comparison procedures.

\section{Method}

In order to carry out the above-mentioned goals, we designed a Monte Carlo type simulation experiment, based on four areas: types of null hypothesis, configuration of the means, number of experimental units within each group ( $N$ $=10,15$, and 19), and degree of correlation $(\rho=.00, .05$, $.10, .15, .20$, and .30$)$ within the groups. The means patterns of the study are from Seaman, Levin, and Serlin (1991), and are shown in Table 1 . Within each pattern the specific values for the means were chosen so that the power for the omnibus $F$ test was approximately of 0.60 for $n=10,0.80$ for $n=15$, and 0.90 for $n=19$, when $a=0.05$ and the variance of each population equals 1 .

The reason we borrowed these mcans patterns is, on the one hand, they are perfectly adapted to the aims of our study, and, on the other, this provided us with an excellent reference frame with which to compare our results (obviously, when the procedures employed coincide and the observations are independent, $\rho_{i}=0$ ). As can be seen the Table 1, when considering the equality of the means, the patterns contain different types of null hypotheses (overail null hypothesis, multiple null hypotheses, and partial null hypothesis) to examine the error rate per comparison. On the other hand, when considering the inequality of the means, the patterns shown in the Table 1 also contain different types of population mean configurations (minimum range configuration, maximum range configuration, and equal space configuration) to examine the per-comparison power.
Table 1

Patterns of Means chosen for the Monte Carlo Study (from Seaman, Levin, \& Serlin, 1991)

\begin{tabular}{ccccc}
\hline Pattern & $\mu_{1}$ & $\mu_{2}$ & $\mu_{3}$ & $\mu_{4}$ \\
\hline 1 & 0.0000 & 0.0000 & 0.0000 & 0.0000 \\
2 & 0.0000 & 0.0000 & 0.0000 & 1.0301 \\
3 & 0.0000 & 0.0000 & 0.8928 & 0.8928 \\
4 & 0.0000 & 0.0000 & 0.5384 & 1.0768 \\
5 & 0.0000 & 0.4097 & 0.4097 & 1.2291 \\
6 & 0.0000 & 0.6313 & 0.6313 & 1.2626 \\
7 & 0.0000 & 0.3993 & 0.7986 & 1.1979 \\
\hline
\end{tabular}

For each of the 126 conditions (7 patterns of means $\times 3$ sample sizes $\times 6$ different correlation structures), 10.000 replications were carried out. Two probabilities were calculated: the error rate per comparison and power per comparison. In the first phase, pseudorandom observation vectors $\mathbf{y}_{\mathrm{i}}=\left[\mathbf{Y}_{\mathrm{i} 1}, \mathbf{Y}_{\mathrm{i} 2}, \ldots, \mathbf{Y}_{\mathrm{i} q}\right]$ with mean vector $\boldsymbol{\mu}^{\prime}=\left[\mu_{1}\right.$, $\left.\mu_{2}, \ldots, \mu_{q}\right]$ and variance-covariance matrix $V$ were generated using the GAUSS generator RNDN (Gauss, 1992). The corresponding observation vectors $\mathbf{y}_{\mathrm{i}}$ were accomplished according to Schauer and Stoller's (1966) method,

$$
\mathbf{y}_{\mathrm{i}}=\boldsymbol{\mu}+\mathbf{T} \mathbf{z}_{\mathbf{i}}
$$

where $\mathbf{T}$ is a lower triangular matrix satisfying the equality $\mathbf{V}=\mathbf{T T}^{\prime}$ and $\mathbf{z}_{\mathrm{i}}$ is an independent normally distributed vector obtained according to the computation method proposed by Kinderman and Ramage (1976).

In the second phase, we proceeded to eliminate the dependence we had previously introduced in the data, according to the procedure developed by Pavur (1988).

Finally, to conduct the above-mentioned calculations, we developed a program in Gauss (v.2.0.) language.

\section{Results}

Within each pattern of means, the Type I error rates were calculated, dividing the number of comparisons falsely declared significant by the total number of comparisons whose means did not differ. To facilitate the interpretation of the empirical results, we present the Type I error rates averaged across nuil and nomull patterns of means for each sample size, significance level, and degree of correlation. Table 1 reveals tive nonnull patterns when the overall null hypothesis is not true, but some population means are equal (partial and multiple null hypotheses). However, whenever an important deviation from the general description (see Tables 2 and 5 ) is associated with a particular pattern, this is pointed out in the text. 


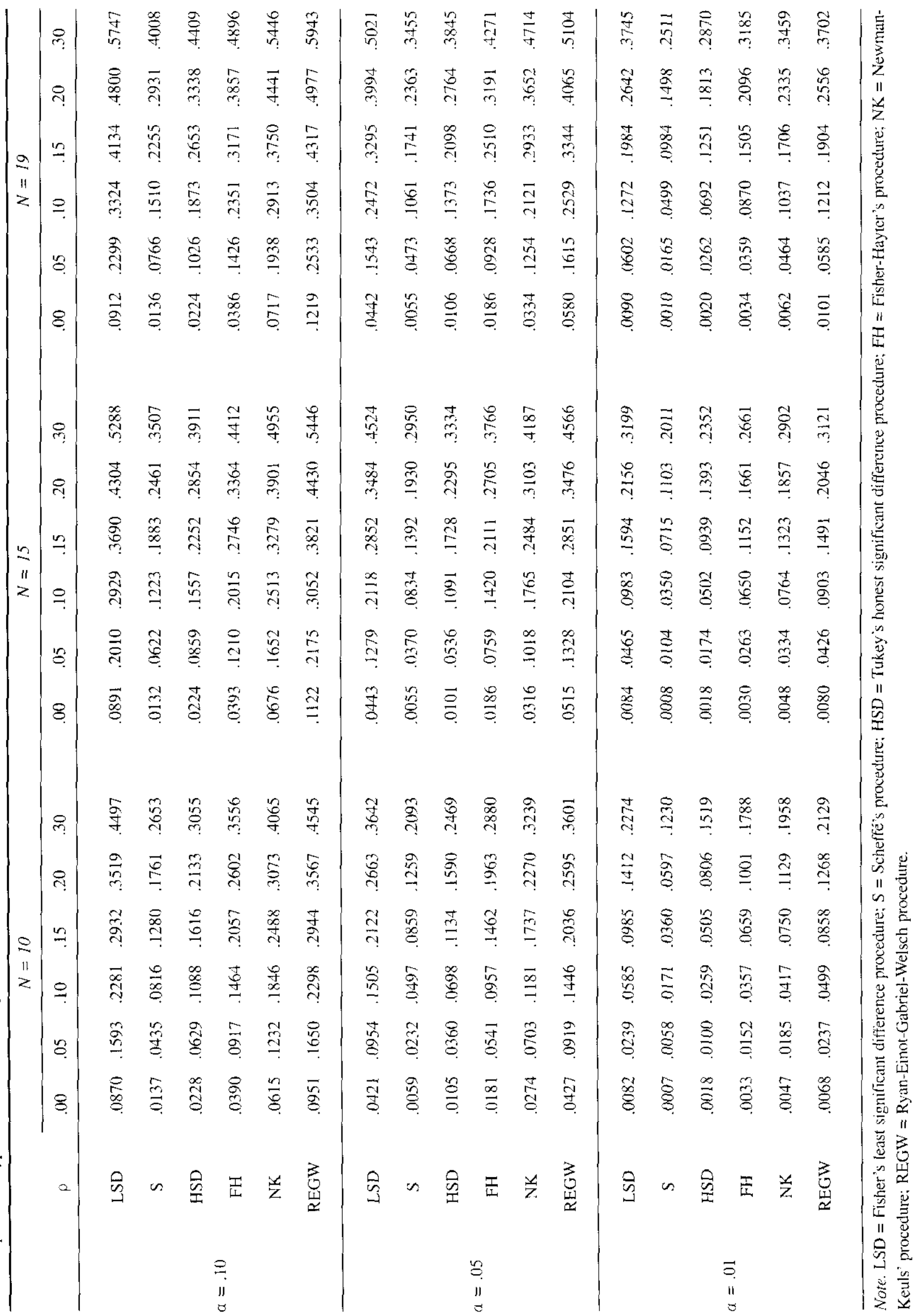




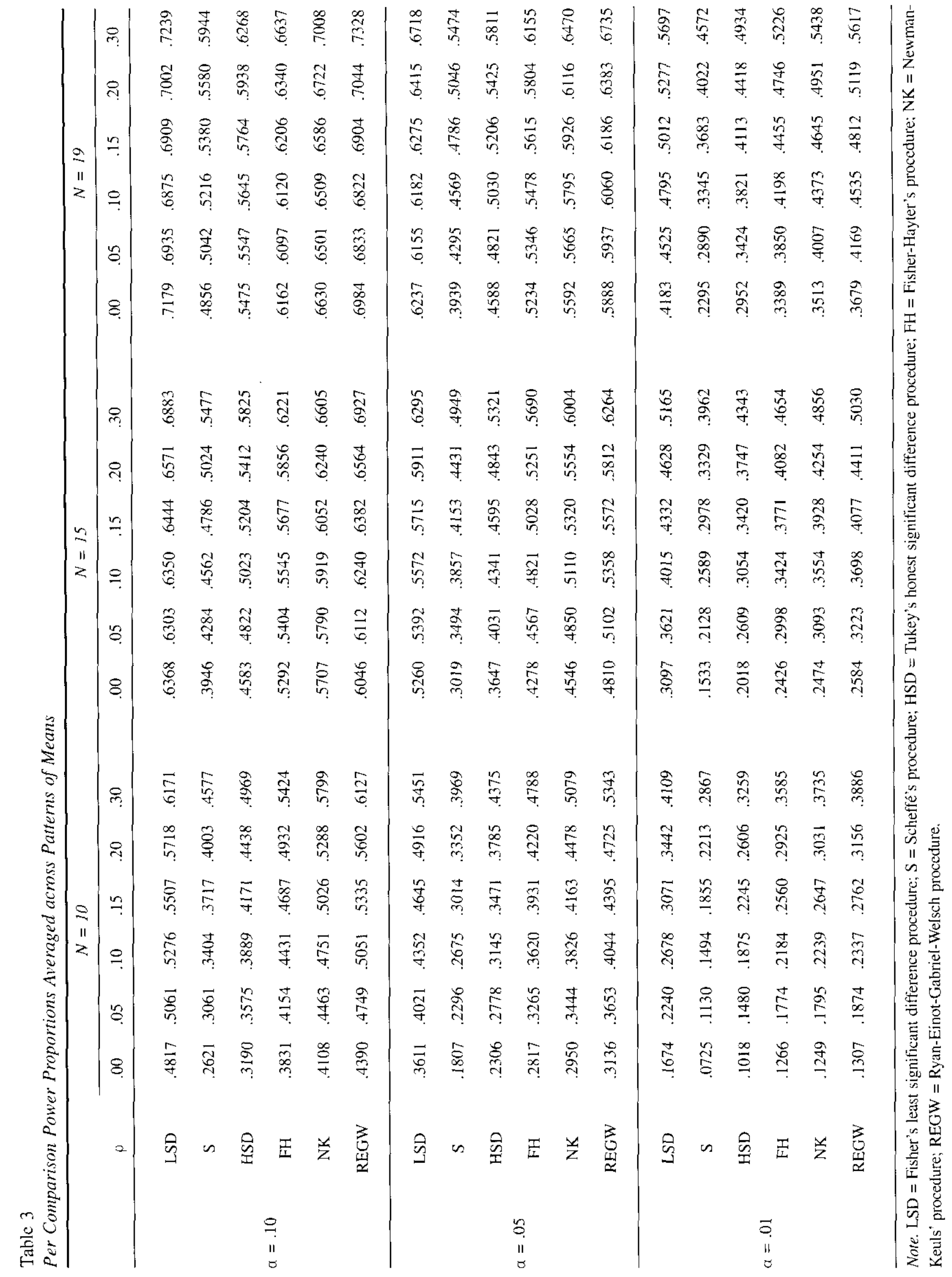


In Table 2, we present the results corresponding to the Type 1 error rate; as can be seen, the dependence produces a large effect in each of the various multiple comparison procedures (MCP), multiplying the per comparison error rate. If we arrange the various multiple comparison procedures based on the error rate, those with the highest error rate are the REGW and LSD procedures, followed by NK, FH, HSD, and $S$. With regard to this sequence, the $\mathrm{FH}$ procedure causes more errors than the $\mathrm{NK}$, in some conditions of the pattern of means I. On the other hand, the LSD procedure makes more errors than the REGW in all the conditions of the pattern of means 1 , and in nearly all those of pattern 2; and the REGW makes a greater number of errors in nearly all the tested conditions of the rest of the patterns of means (for conditions 3 to 6 , see Table 1).

The per-comparison power rates were calculated, dividing the number of comparisons falsely declared nonsignificant by all pairs of unequal means in the pattern. Table 1 shows that, although each one of the nonnull patterns of means was examined for the same effect sjze, within each pattern the different procedures can be evaluated as a function of the differences betwoen the means. When this was taken into account, besides verifying that the larger the eflect size the more powerful were the procedures, we also observed that the procedures did not behave uniformly along the various sizes. For example, when the means were equally spaced and $n=10$, the FH procedure was more powerful than the NK procedure for effect size 0.8 ; but it was less powerful for the remaining effect sizes. For each and every one of the patterns, the per-comparison power rates averaged through the different effect sizes were always observed to be larger for the sequential procedures than for the simulaneous procedures. As this discovery was consistent for all the conditions we manipulated in our study, we considered it appropriate to condense the information corresponding to the types of the population mean configurations (see Tables 3 and 6) and to point out the most relevant deviations in the text.
The effects of the comelation are different for the per comparison power (see Table 3). But, as in the previous case, an increase in the correlation is almost always accompanicd by an increase in the power; when the latter is high, an increase in dependence produces a decrease in the power. If we arrange the various multiple comparison procedures from greater to lesser power, we would obtain the following sequence: LSD, REGW, NK, FH, HSD, and S. In some cases (with a high degree of dependence) the NK procedure shows more power; the situations in which this occurs show low correlation and a Iow significance level.

One of the working hypotheses of this investigation was to see whether the different multiple comparison procedures, in the same pattern of means, reach their minimal and maximum values in the per comparison error rate and power. The results obtained, presented in Table 4, show that in the case of the power, all the MCP obtain their maximum value in pattern 2 and the minimum value in pattern 7. However, we did not find this uniformity in the case of the Type I error rate, where the maximum values were found in patterns 3 (HSD, NK, and REGW), 4 (LSD and FH), and 6 (S) and the minimum values were located in palterns 1 (LSD, $\mathrm{FH}$, NK. and REGW) and 5 (S and HSD).

\section{Results with corrected data}

In Table 5, we present the corresponding results of the Type I error rate, once the dependence of the data was corrected with the procedure described by Pavur (1988). In the LSD procedure by Fisher (1935,) the correction maintained the Type I crror rate at the chosen nominal level. In other procedures, such as $\mathrm{S}, \mathrm{HSD}$, and $\mathrm{FH}$, the alteration matintained the error rate at the same level as when the dependence was nul]. In the two remaining procedures. NK and REGW, the error rate was reduced when data were corrected. In pattern 1 , the FH procedure presented a higher error rate when the dependence was greater, and the NK

Table 4

Minimum and Maximum Values of the various Multiple Comparison Procedures in the Type I Error Rate and Power

\begin{tabular}{|c|c|c|c|c|c|c|c|c|}
\hline & \multicolumn{4}{|c|}{ Type I Error } & \multicolumn{4}{|c|}{ Power } \\
\hline & Maximun & Pattern & Minimum & Pattern & Miximum & Pattern & Minimum & Pattern \\
\hline LSD & .0535 & 4 & .0195 & 1 & .7236 & 2 & .4069 & 7 \\
\hline $\mathrm{S}$ & .0062 & 6 & .0042 & 5 & .4806 & 2 & .2180 & 7 \\
\hline ISSD & $.01 ! 4$ & 3 & .0087 & 5 & .5647 & 2 & .2665 & 7 \\
\hline $\mathrm{FH}$ & .0213 & 4 & .0133 & 1 & .6396 & 2 & .3170 & 7 \\
\hline NK & .0450 & 3 & .0126 & 1 & .6710 & 2 & .3423 & 7 \\
\hline REGW & .0813 & 3 & .0138 & 1 & .6919 & 2 & .3731 & 7 \\
\hline
\end{tabular}

Note, LSD = Fisher's least significant difference procedure; $S=$ Scheffés procedure: $H S D=$ Tukey's honest significant difference procedure; FH = Fisher-Hayter's procedure; NK = Newman-Keuls' procedure; REGW = Ryan-Einot-Gabriel-Welsch procedure. 


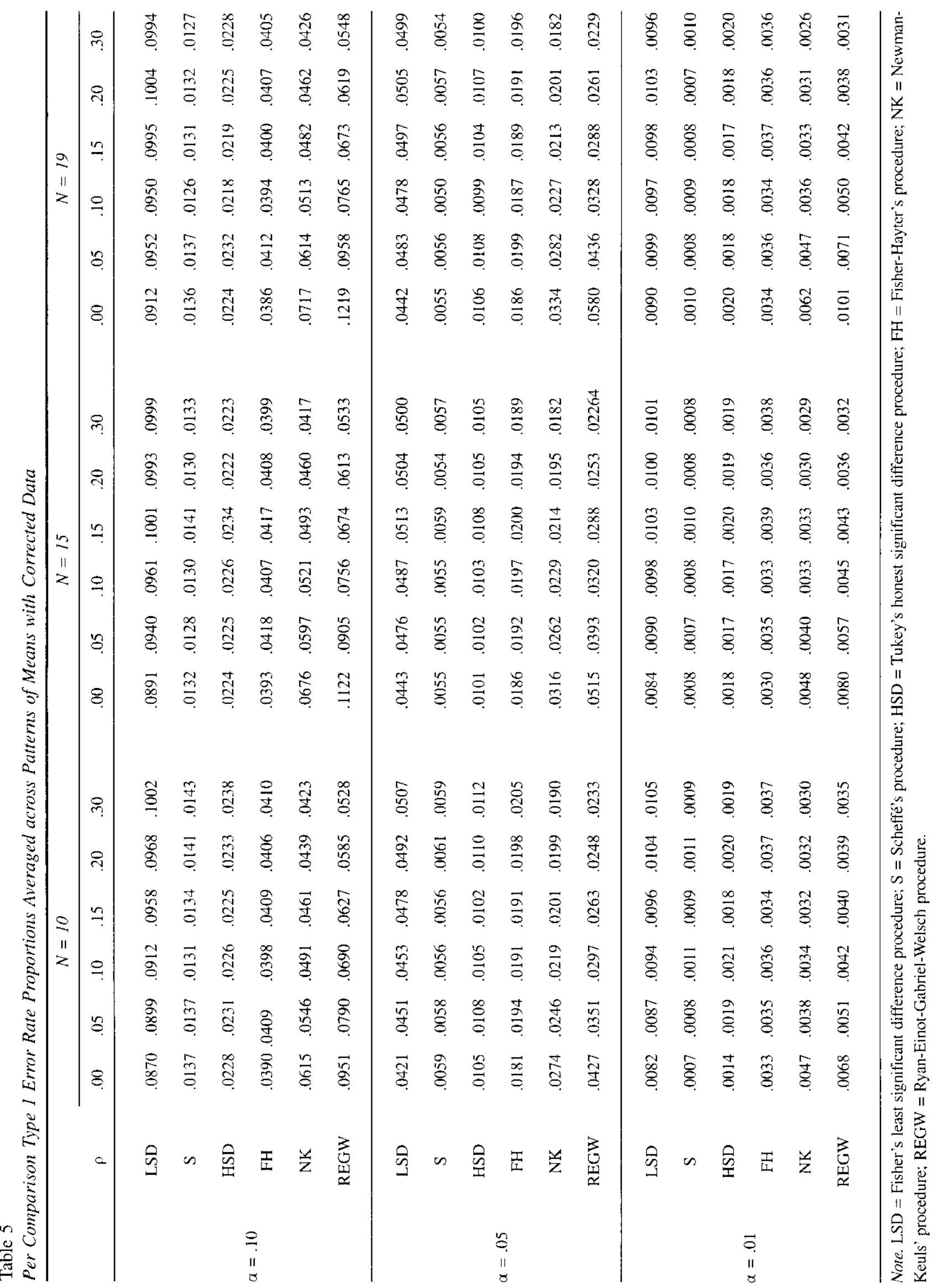




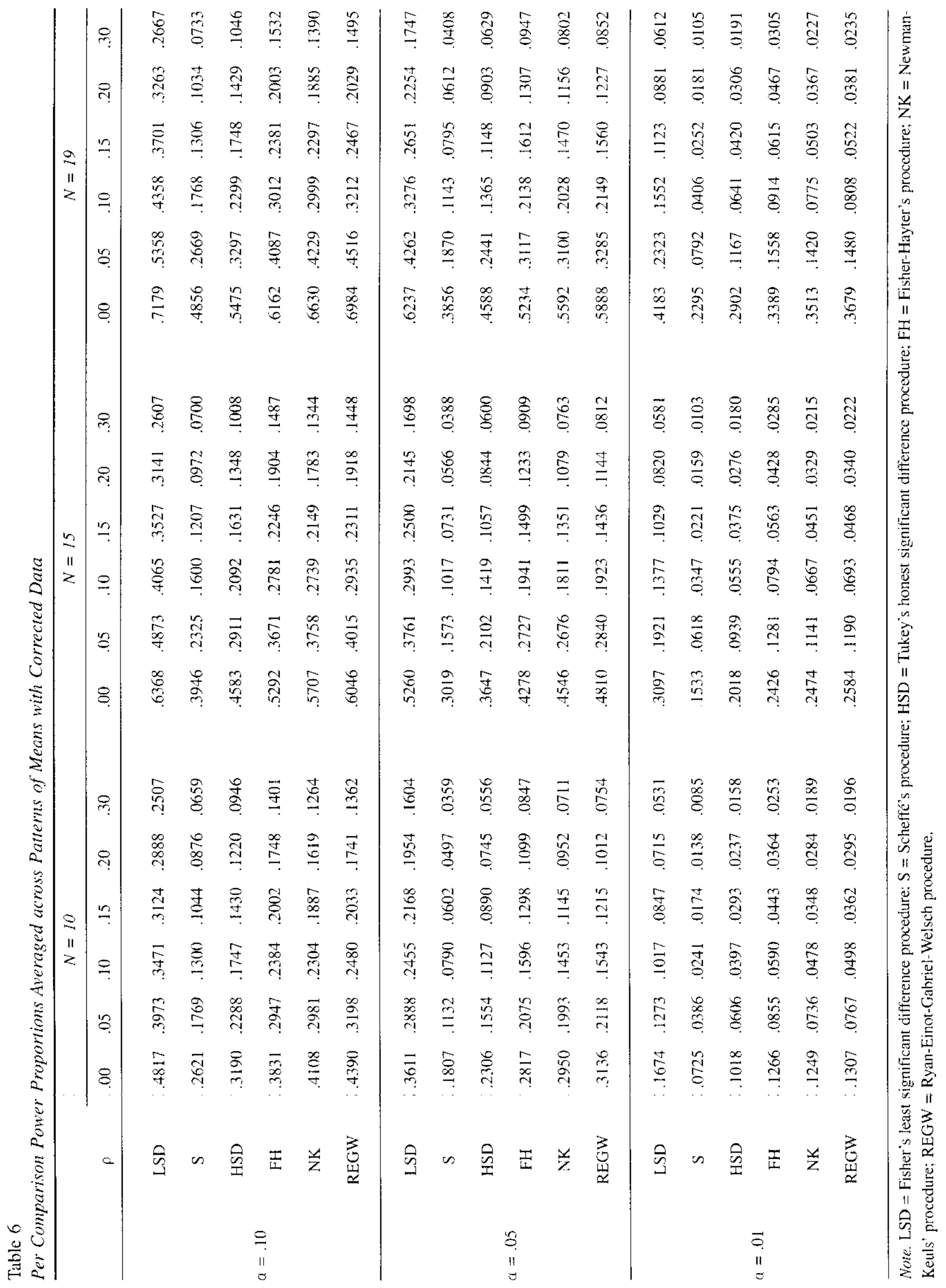


and REGW procedures were maintained at the same level as when the correlation was zero. When dependence was increased, the $\mathrm{FH}$ procedure showed a large decrease in the error rate in patterns 3,5 , and 6 , and a slight decrease in patterns 2 and 4 . The REGW procedure showed a slight decrease in pattern 2 only, and a notable decrease in patterns $3,4,5$, and 6 .

Table 6 shows the results of the per comparison power of the various procedures, once the dependence has been corrected. As the dependence decreased, the power was also reduced, and this reduction was greater when the initial correlation of the data was higher.

\section{Discussion}

We would like to compare the results obtained in this study with those obtained by other authors, using the same variables.

Our results coincide with those obtained by Scariano and Davenport (1987), insofar as the ANOVA is affected by the dependence of the data. We also coincide with the results published by Seaman, Levin, and Serlin (1991) where various multiple comparison procedures across several patterns of means were used, with independent data, arriving at the same results in those situations that are identical in both investigations.

Finally, we wish to compare our results with those found by Vallejo and Menendez (1995). The latter study was carried out with three groups $(q=3)$, whereas the current one used four $(q=4)$. For the multiple comparison procedures, the results are similar in both studies, but we would like to make some clarifications. When using dependent data, slightly lower values were obtained in the $q=4$ condition than in the $q=3$, except for the cases referred to in Type I error rate, which were slightly higher, using the LSD and REGW procedures. Upon introducing the correction, the results were similar, obtaining lower values in $q=4$, except for two cases where the value was slightly higher in both rates (Type I crror and power), both using the ISSD procedure. The data correction did not cause much difference between the studies, the Type I error rate was similar in all the procedures, except the L.SD and FH, where, instead of decreasing slightly $(q=3)$, it increased slightly $(q=4)$. Regarding the power, when the correlation was corrected, we found a similar operation in all the procedures, except for the LSD which was supcrior for $q$ $=4$, because the decline was smaller; as was the loss of power.

In summary, the presence of the correlation in our data has a very negative effect on the data, in view of the poor results obtained when correcting this undesired effect. In short, when using dependent data, more Type I errors are committed; if dependence is corrected, then the power is reduced.

\section{References}

Aitken, A.C. (1935). On least squares and linear combination of observation. Proceeding of the Royal Society of Edinburgh, 55, 42-48.

Einot. I., \& Gabriel, K.R. (1975). A study of the power of several methods of multiple comparisons. Joumal of the American Statistical Association. 70, 574-583.

Fisher, R.A. (1935). The Design of Experiments. Edinburgh and London: Oliver and Boyd.

GAUSS (1992). The Gatss System (version 3.1). Washington: Aptech Systems, Inc.

Hayter. A.J. (1986). The maxinum fannilywise error rate of Fisher's least significant difference test. Joumal of the American Statistical Association, 81, 1000-1004.

Keuls, M. (1952). The use of the 'Studentized range' in connection with and analysis of variance. Euphtyca, 1, 112-122.

Kinderman, A.J., \& Ramage, J.G. (1976). Computer generation of normal random numbers. Jormal of American Statissical Association, 77, 893-896.

Newman. D. (1939). The distribution of the range in samples from a normal population expressed in terms of an independent estimate of standard deviation. Biometrika, 3I, 20-30.

Pavur, R. (1988). Type I error rates for multiple compitrison procedures with dependent data. American Statistician, 42, $171-174$.

Pavur, R., \& Lewis, T.D. (1983). Unbiased $F$ test for factorial experiments for cortelated data. Communications in Statisics, Theor, and Methods, 12, 829-840.

Ryan, T.A. (1959). Multiple comparisons in psychological research. Psychological Bulletin, 56, 26447.

Scariano, S.M., \& Davenport, J.M. (1987). The effects of violations of independence assumptions in the one-way ANOVA. American Statistician, 41, 123-129.

Schaffer, J.P. (1995). Multiple hypothesis testing. Annual Review Psychology, 46, 561-584.

Schauer, E.M., \& Stoller, D.S. (1966). On the generation of normal random vectors. Technonetrics, 4, 451-464,

Scheffé, H. (1959). The Analysis of Variance, New York: Wiley.

Seaman, M.A., Levin, J.R., \& Serlin, R.C. (1991). New developments in pairwise multiple comparisons: Some powertul and practicable procedures. Psychological Balletin, 110, 577-586

Tukey, V.W. (1953). The Problem of Multiple Comparisons. Mimeographed Monograph, Princeton University.

Vallejo, G.. \& Fernández, P. (1990). Diseños de medidas repetidas con errores autocorrelacionados. Psicothema, 2, 189-209.

Vallejo, G., \& Menéndez, I.A. (1995). Comparaciones múltiples en diseños transversales con datos dependientes. Psicothema, 7. $401-418$

Welsch, R.E. (1977). Stepwise nultiple comparison procedures. Iournal of the American Statistical Association, 72, 566-575.

Received October 21, 1997

Revision received April 5, 1998 Accepted October 20, 1998 